GA-A22138

\title{
BOLOMETRY FOR DIVERTOR CHARACTERIZATION AND CONTROL
}

\author{
FFR 07 1945
}

by OSTI

A.W. LEONARD, J. GOETZ, C. FUCHS, M. MARASHEK,

F. MAST, and R. REICHLE

OCTOBER 1995 


\section{DISCLAIMER}

This report was prepared as an account of work sponsored by an agency of the United States Government. Neither the United States Government nor any agency thereof, nor any of their employees, makes any warranty, express or implied, or assumes any legal liability or responsibility for the accuracy, completeness, or usefulness of any information, apparatus, produce, or process disclosed, or represents that its use would not infringe privately owned rights. Reference herein to any specific commercial product, process, or service by trade name, trademark, manufacturer, or otherwise, does not necessarily constitute or imply its endorsement, recommendation, or favoring by the United States Government or any agency thereof. The views and opinions of authors expressed herein do not necessarily state or reflect those of the United States Government or any agency thereof. 


\title{
BOLOMETRY FOR DIVERTOR CHARACTERIZATION AND CONTROL
}

\author{
by \\ A.W. LEONARD, J. GOETZ,† C. FUCHS, ¥ M. MARASHEK, $\ddagger$ \\ F. MAST, $\neq$ and R. REICHLE $\triangle$
}

This is a preprint of a paper to be presented at the Diagnostics for ITER International Workshop, August 28-September 1, 1995, Varenna, Italy, and to be printed in the Proceedings.

Work supported by

U.S. Department of Energy

Contract No. DE-AC03-89ER51114

†Massachusetts Institute of Technology

ҒMax Planck Institute

$\Delta$ Joint European Torus

GENERAL ATOMICS PROJECT 3466 OCTOBER 1995 


\title{
BOLOMETRY FOR DIVERTOR CHARACTERIZATION AND CONTROL
}

\author{
A.W. Leonard, ${ }^{1}$ J. Goetz, ${ }^{2}$ C. Fuchs, ${ }^{3}$ M. Marashek, ${ }^{3}$ F. Mast,${ }^{3}$ \\ R. Reichle ${ }^{4}$ \\ $1_{1}$ General Atomics, P.O. Box 85608, San Diego, CA 92186-9784 \\ 2Massachusetts Institute of Technology \\ ${ }^{3}$ Max Planck Insitute \\ 4Joint European Torus
}

\section{INTRODUCTION}

Operation of the divertor will provide one of the greatest challenges for ITER. 1 Up to $400 \mathrm{MW}$ of power is expected to be produced in the core plasma which must then be handled by plasma facing components. Power flowing across the separatrix and into the scrape-off-layer (SOL) can lead to a heat flux in the divertor of $\approx 30 \mathrm{MW} / \mathrm{m}^{2}$ if nothing is done to dissipate the power. This peak heat flux must be reduced to $\sim 5 \mathrm{MW} / \mathrm{m}^{2}$ for an acceptable engineering design. The current plan is to use impurity radiation and other atomic processes from intrinsic or injected impurities to spread out the power onto the first wall and divertor chamber walls. It is estimated that $300 \mathrm{MW}$ of radiation in the divertor and SOL will be necessary to achieve this solution.

Measurement of the magnitude and distribution of this radiated power with bolometry will be important for understanding and controlling the ITER divertor. Shown in Fig. 1 is a sketch of the ITER divertor with possible regions of strong radiation. Present experiments have shown intense regions of radiation both in the divertor near the separatrix and in the X-point region. The task of a divertor bolometer system will be to measure the distribution and magnitude of this radiation.

The bolometric measurements will have a number of uses. First, radiation measurements can be used for machine protection. Intense divertor radiation will heat plasma facing surfaces that are not in direct view of temperature monitors. Measurement of the radiation distribution will provide information about the power flux to these components. Secondly, a bolometer diagnostic is a basic tool for divertor characterization and understanding. Radiation measurements are important for power accounting, as a cross check for other power diagnostics, and gross characterisation of the plasma behavior. A divertor bolometer system can provide a 2-D measurement of the radiation profile for comparison with theory and modeling. Finally a bolometer system can provide realtime signals for control of the divertor operation. Important characteristics for control might include the magnitude of $\mathrm{X}$-point radiation, the balance of radiation in the inboard and outboard divertors, or the location above the divertor floor of the most intense radiation.

Specifications for measurement of divertor radiation to meet the above goals has been put together by the ITER diagnostic group. ${ }^{2}$ They are listed in Table 1.

Table 1. Specifications for measurement of divertor radiation.

\begin{tabular}{lllll}
\hline Total Radiated Power & Max. Amplitude & Time Response & Accuracy & \\
\hline X-point region & $\leq 0.6 \mathrm{GW}$ & $10 \mathrm{~ms}$ & $\pm 10 \%$ & \\
Divertor & $\leq 0.6 \mathrm{GW}$ & $10 \mathrm{~ms}$ & $\pm 10 \%$ & \\
Radiation Profile & Max. Amplitude & Spatial Resolution & Time Response & Accuracy \\
\hline X-point & $\leq 300 \mathrm{MWm}^{-3}$ & $20 \mathrm{~cm}$ & $10 \mathrm{~ms}$ & $\pm 20 \%$ \\
Divertor & $\leq 00 \mathrm{MWm}^{-3}$ & $5 \mathrm{~cm}$ & $10 \mathrm{~ms}$ & $\pm 30 \%$ \\
\hline
\end{tabular}




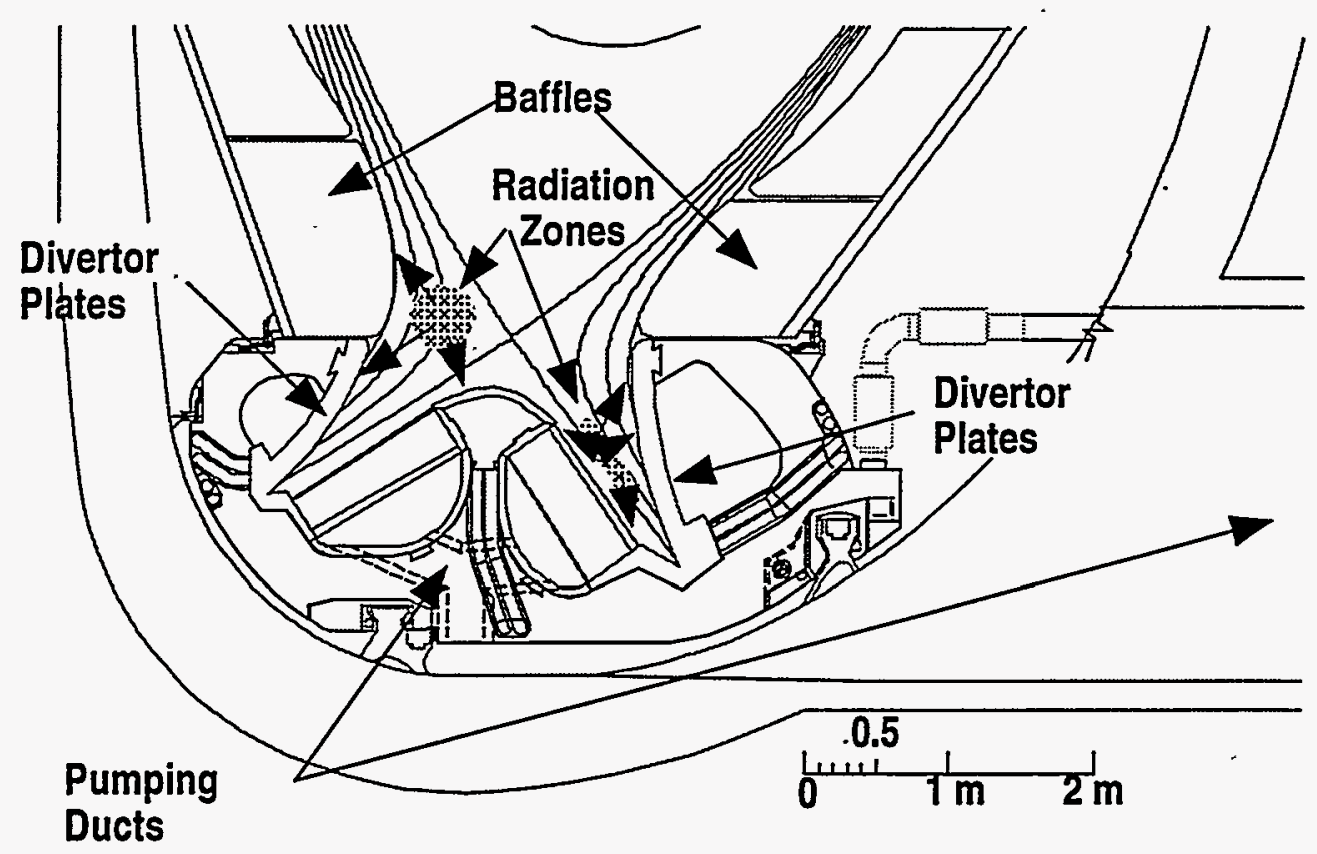

Fig. 1. A sketch of the ITER divertor. Shown are the vertical target plates, the private flux structure and possible regions of intense radiation.

The desired resolution of emission intensity has not been specified, but given experience with current divertor experiments a line-averaged emissivity of $0.1 \mathrm{MWm}^{-3}$ should be resolved. The specification for $5 \mathrm{~cm}$ resolution in the divertor is driven by the desire for radial profiles in the divertor leg. For gradients parallel to the poloidal field, the $20 \mathrm{~cm}$ specification may be adequate.

For this discussion of divertor bolometry for ITER, a description of bolometer systems currently in use on the world's major tokamaks will first be presented in Section II. This description will serve to highlight techniques and limitations of extracting 2-D information from a limited number of views. A discussion of applying these techniques to ITER will be presented in Section III with a summary and conclusions in Section V.

\section{BOLOMETER SYSTEMS CURRENTLY IN USE}

Bolometer systems for measuring divertor radiation are currently in use on the tokamaks ASDEX-U and JET in Europe, ALCATOR-CMOD and DIII-D in the U.S. and JT-60U in Japan. All these systems are based on detectors consisting of small-sized platinum or gold resistors that change resistance as they are heated by plasma radiation. ${ }^{3}$ The detector power is then calculated using the temperature measurement and the detector thermal characteristics. A 2-D emissivity profile can then be calculated by careful analysis of all the detector signal levels.

The common difficulty with inverting the measured signals into a 2-D poloidal profile is the limited number of views of these systems. Consider a divertor region that is partitioned into the desired resolution of $m \times n$ cells. Typically only two or three cameras, each with $\mathrm{n}$ chords covering the region, have been installed for this measurement. However, $m$ cameras with distinct views are needed to unambiguously specify the emissivity in each cell. The inversion of the measurements into a radiation profile is given by the following equation:

$$
\bar{S}_{i}=\bar{T} \bar{I}_{m \times n}
$$

where $S$ is a vector of $i$ signals, $I$ is the vector of $m \times n$ cells and $T$ is a transformation matrix containing the $i$ th sensor's response to a unit of radiation in the $m, n$th cell. The elements of $S$ and $\mathrm{T}$ are known and the matrix $\mathrm{T}$ must be inverted to solve for $\mathrm{I}$, the radiation distribution. When the number of radiating cells, $m \times n$, greatly outnumbers the number of measurements, $i$, the matrix $T$ is very singular and cannot, in general, be inverted without additional constraint equations. Each divertor program has developed individual methods to handle this inversion problem. Three divertor bolometer systems and their associated analysis will be briefly described below to illustrate what information can be extracted from a given set of chords.

An example of a divertor bolometer system is that of ALCATOR-CMOD 4 shown in Fig. 2. This system consists of three cameras with four viewing chords each. The cameras are well 


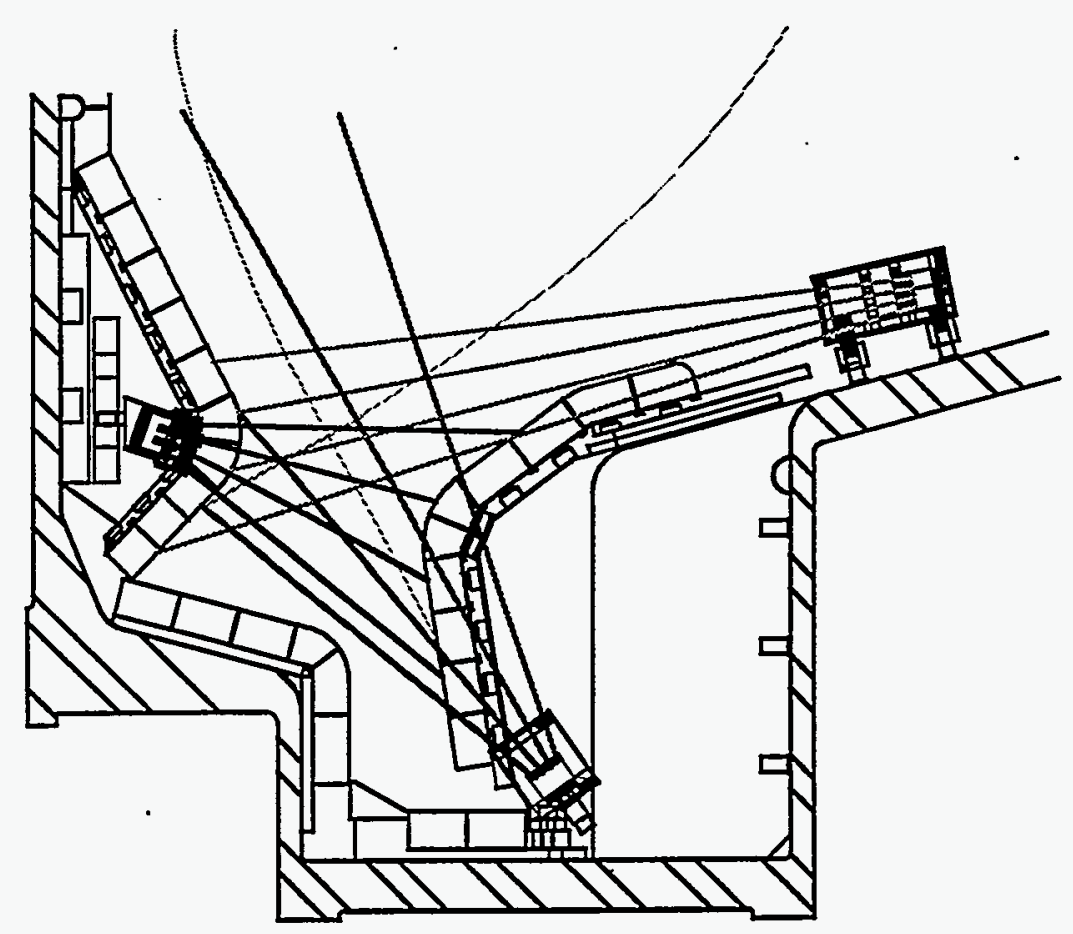

Fig. 2. The geometry of the ALCATOR-CMOD divertor bolometry system. Three bolometer cameras are each installed with four detectors.

separated poloidally to acquire unique information. Before inverting the divertor radiation, the core plasma radiation is measured by a midplane system and subtracted from those divertor chords which also view the main plasma. The divertor region is divided into a $9 \times 12$ grid, $2 \mathrm{~cm}$ a side for each cell and additional smoothing equations are added to the transformation equation, Eq. (1), to make the matrix $\mathrm{T}$ less singular. The smoothing equations effectively limit the second derivative of the radiation profile. Finally the matrix is inverted using singular value decomposition (SVD) techniques. 5 Matrix inversion with SVD is useful for inverting near singular matrixes and extracting the principle values.

An example of a radiation profile produced by the ALCATOR-CMOD bolometer system and subsequent analysis is shown in Fig. 3. The inversion sets the emissivity level in the center of the cell, but for this figure the contour levels are interpolated between cell centers. This example of a highly radiating divertor illustrates the detail that can be extracted from the data set. The greatest emissivity is seen in both divertors near the strike point locations. In the outboard divertor strikepoint emissivity can be distinguished from emission near the $\mathrm{X}$-point, but not details of the radial profile from the separatrix.

Another example of a bolometer system is that of DIII-D, ${ }^{6}$ shown in Fig. 4. Here there are two cameras each of which spans the main plasma and the divertor region. A total of 10-12 of the 48 channels view the divertor plasma, similar to that for ALCATOR-CMOD. To obtain the divertor radiation profile the contributions from the main plasma radiation must first be subtracted from the divertor chords of the upper camera. The main plasma radiation is assumed constant on a flux surface and is fit to a spline function with bolometer channels that do not view the divertor. Additional constraint equations are then added to the divertor radiation signals in vector $S$ of Eq. (1). For DIII-D the divertor radiation is described by a 2-D spline function of the magnetic flux surface and the height $z$ above the divertor plate. With this constraint, plasma radiation can be limited to the SOL region where significant power is flowing, excluding radiation from the private flux region and far SOL. Such constraints are necessary for the DIII-D geometry with only two poloidal views. The spline function adds a series of nonlinear equations that have not yet been put into the form of matrix Eq. (1). To obtain the radiation profile, the spline parameters are adjusted through iteration to minimize the error given by:

$$
\chi^{2}=\sum_{i}^{l} \frac{\left(S_{i}-M_{i}\right)^{2}}{(l-h)\left(\eta_{i} S_{i}\right)^{2}}
$$




\section{HIGHLY RADIATIVE DIVERTOR}
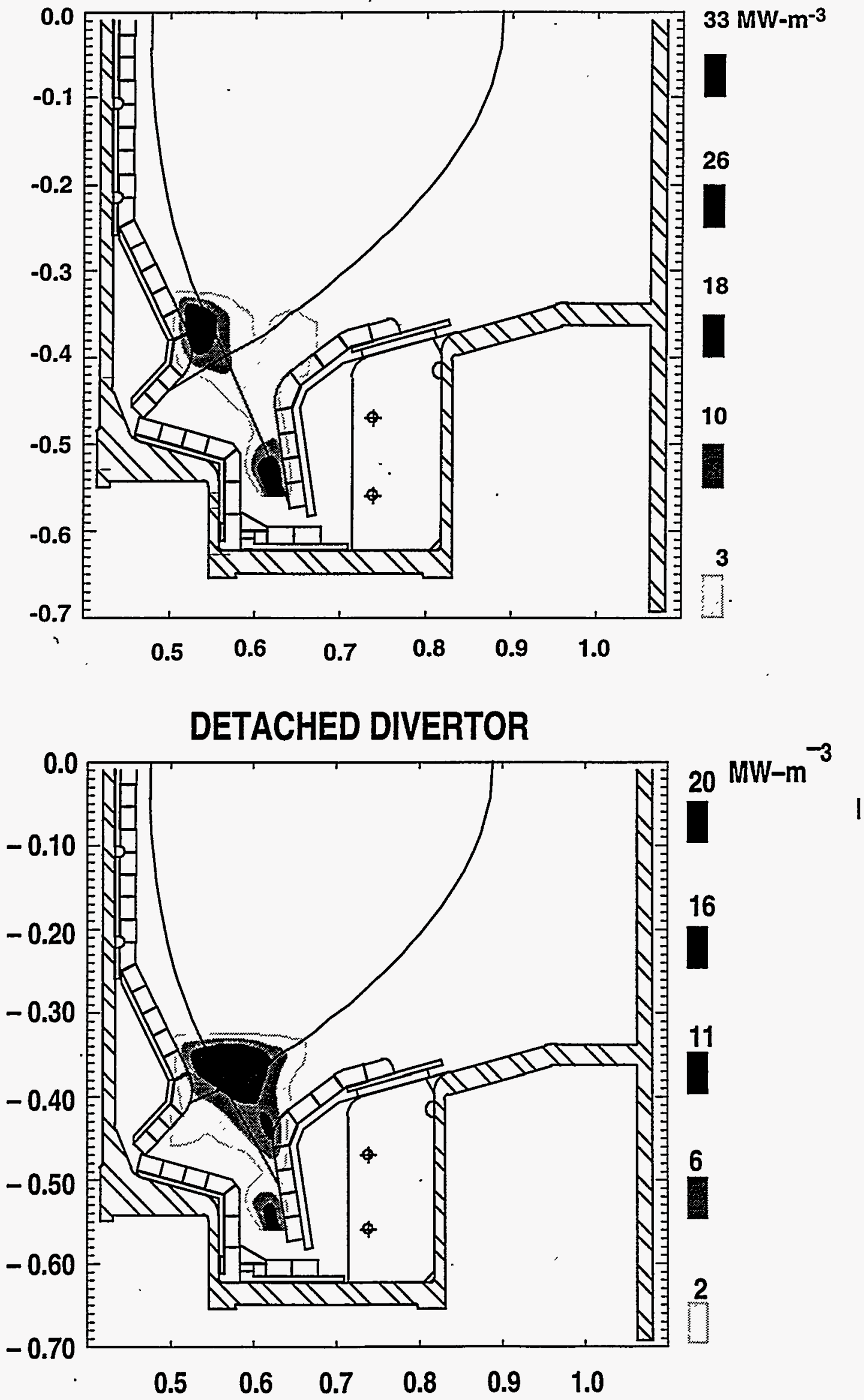

Fig. 3. A radiation profile obtained from inversion of ALCATOR-CMOD bolometry data. 
where $S_{i}$ is the measured signal and $M_{i}$ is signal that would be obtained with the fitted profile and $\eta$ is the uncertainty in the measurement. The number of spline parameters is given by the variable h.

An example of a radiation profile obtained by this procedure is shown in Fig. 5. The scale of the resolved features are similar to that for the ALCATOR-CMOD example. In this case, the strong radiation can be seen extending from the $\mathrm{X}$-point region to the strike-point in the outboard divertor. Once again radiation from the $\mathrm{X}$-point can be distinguished from the strike-point, but the radial resolution from the separatrix is limited. This would be expected from the chord geometry of Fig. 4. From the side camera there are three chords which intersect the outboard divertor, but the SOL is only as wide as one of the upper camera chords.

A final example is the bolometer system of ASDEX-U7 shown in Fig. 6 . A total of 72 channels are now operational in five cameras. The 40 channels of horizontal cameras span the main chamber and divertor region as do the 24 channels of the upper vertical camera. An additional eight channels viewing the lower divertor with a finer spatial resolution have been installed but are not yet operational. For the inversion of the ASDEX-U data additional constraint equations are also added to the transformation Eq. (1). In this case the second derivative of the radiative solution is limited, but by different amounts in the direction perpendicular and parallel to the poloidal field lines. This smoothing constraint is modelled as a diffusion equation. By adjusting the "diffusion" constants one can obtain a solution free of numerical artifacts yet with an acceptable fit to the data. The solution is then obtained by minimizing the following function:

$$
\mathfrak{S}_{\text {min }}=\int(\nabla \cdot(D \nabla I))+A \sum_{i}^{l} \frac{\left(S_{i}-M_{i}\right)^{2}}{\left(\eta S_{i}\right)^{2}}
$$

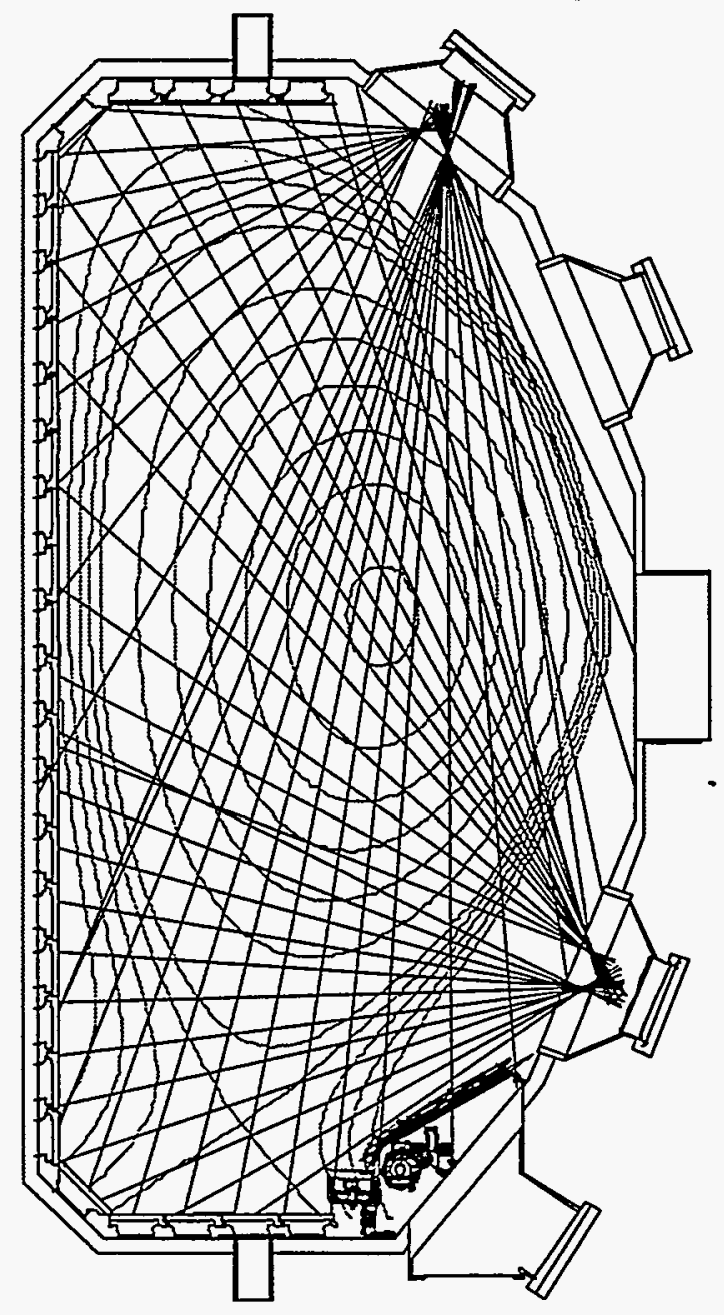

Fig. 4. The bolometer system on DIII-D contains two cameras each with 24 channels. The view of each camera spans the entire plasma with finer resolution of the divertor regions. 


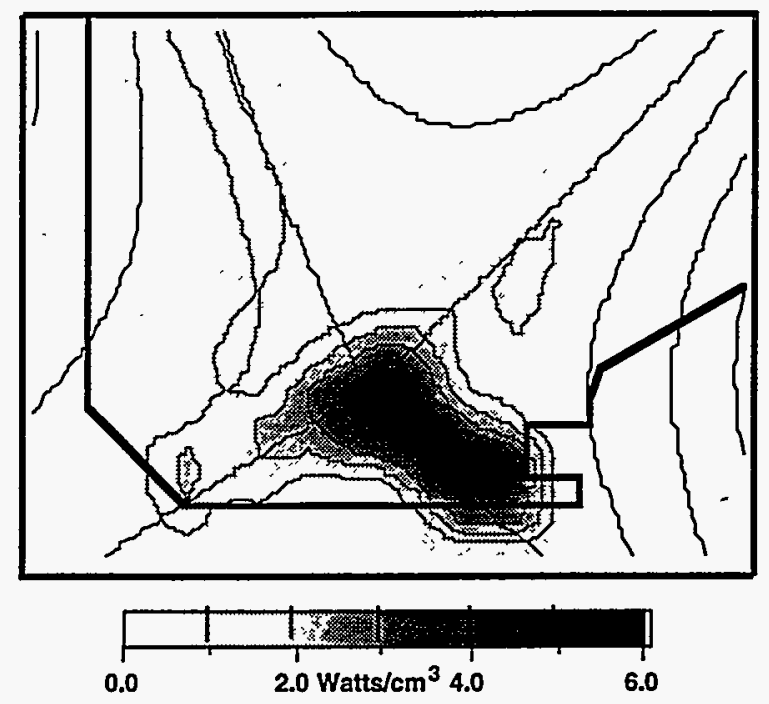

Fig. 5. An inversion of DIII-D bolometry data shows radiation extending from the $\mathrm{X}$-point to the divertor floor during radiative divertor experiments.

where $D$ is the anisotropic "diffusion" tensor, and again $S_{i}$ is the actual detector signals and $M_{i}$ is the fitted signal due to radiation profile $I$. The variable A determines the trade-off between the smoothness of the solutions and the agreement of the fit to the data. By using finite differences for the gradient in the radiation profile a linear system of equations is obtained that can be solved numerically. For ASDEX-U the entire radiation profile, the main plasma and divertor is solved in this manner simultaneously.

An example of a radiation profile obtained for ASDEX-U is shown in Fig. 7. This example is a highly radiating plasma produced by neon injection. Finer scale resolution in the divertor is more difficult because the views of the midplane and upper cameras are becoming more parallel in the divertor region. The lower horizontal camera will be of significant help when it becomes operational.

The ASDEX-U program has also done additional work on using the bolometer signals for real-time control of impurity gas puffing for divertor experiments. ${ }^{8}$ For these experiments a realtime signal of the main plasma radiation was desired. Because hardware was available for only 10 channels of real-time processing an SVD analysis was performed on the bolometer data from a range of relevant plasmas to determine the most appropriate channels. Finally the main plasma radiation was reconstructed with all the available channels from which a linear regression analysis was performed to determine the best linear combination of the chosen channels to represent the total main chamber radiation. This method has produced a sufficiently accurate real-time signal of main chamber radiation for control of impurity gas injection for enhanced radiation experiments. It is easy to conceive of using a similar method for real-time signals of the divertor radiation profile.

\section{DIVERTOR BOLOMETRY FOR ITER}

Using the systems described above as a guide one can produce a conceptual design for an ITER bolometer system. The basic goal will be to cover all plasma of the divertor and $\mathrm{X}$-point region with at least three views from well separated cameras. The chord spacing within each camera should be such as to achieve the desired resolution. A sketch of such a system is shown in Fig. 8. This concept includes six cameras for each divertor, three viewing from the private flux region and three from the outside the vertical divertor plate. To achieve the desired spatial resolution each camera would need approximately 20 detecting channels.

From the simple sketch of Fig. 8 several design issues become apparent. The bolometer cameras are mounted into the divertor cassette structure itself. The divertor design should include apertures for the bolometer camera views as well as mounting structure and signal cable routing. This is likely the case for other divertor diagnostics as well. The divertor cameras are mounted close to the divertor plasma for two reasons. The first, and most important, is to obtain complete divertor plasma coverage. By bringing the defining aperture of the camera closer to the plasma a much greater field of view of the plasma can be obtained with a smaller opening in the divertor structure. The second reason is that close to the plasma a greater solid angle, and larger signal, is possible for a given spatial resolution. For these reasons it is important to integrate the bolometer diagnostic into the divertor design. 


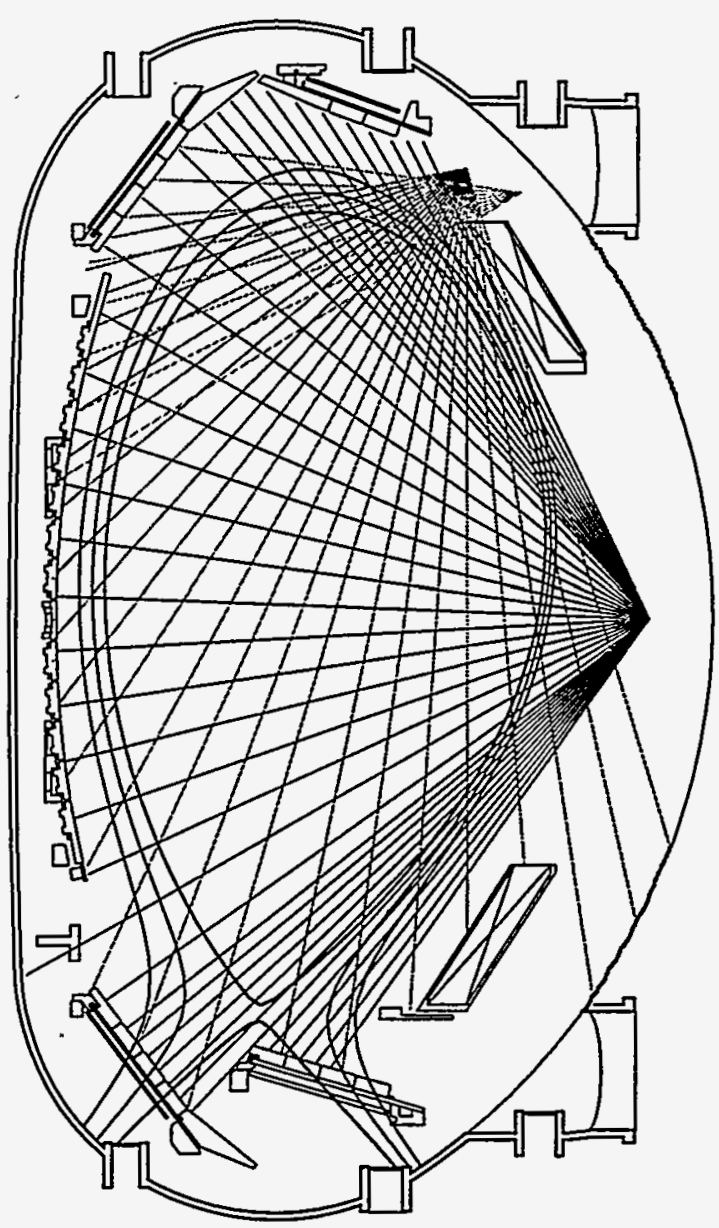

Fig. 6. The operational bolometer cameras on ASDEX-U contain a total of 72 channels in three cameras.

Design of the bolometer cameras into the vertical target plate appears difficult. The vertical plate is a high heat flux component taking direct heat flux from the divertor plasma. The edges of any holes in the surface of this plate would be preferentially heated to intolerable levels. A small gap between divertor cassettes may be sufficient for the bolometer view. The views through the vertical plate are most important for measuring the radial radiation profile. Probably only the profile parallel to the poloidal field can be measured if cameras are installed in the private flux region only. Another possible difficulty is providing apertures in the divertor dome. This structure is designed to take significant heat flux and provide neutron shielding. A camera in this region is important for measuring radiation in the upper divertor and X-point region. A study of the feasibility of such placement should be incorporated into the divertor design.

From the bolometer concept outlined above, one can estimate the signal levels that would be detected. The power onto a detector is given approximately by:

$$
P_{d} \approx \frac{\varepsilon A_{p}}{4 \pi L^{2}}
$$

where $P_{d}$ is the power per unit area on the detector, $A_{p}$ is the area of the viewing aperture, $L$ is the distance of the detector to the aperture and $\varepsilon$ is the line integral of the plasma radiation in power per unit area. If the spacing between detectors arranged behind the aperture is $-2 \mathrm{~cm}$, the detector to aperture radius, $L$, will be about $25 \mathrm{~cm}$ if the spatial resolution of $5 \mathrm{~cm}$ is kept over the bulk of the divertor plasma. The poloidal width of the aperture is set to keep the chords of neighboring channels just overlapping. That would be an aperture of $2 \mathrm{~cm}$ in this example. The toroidal extent of the aperture is only limited by the solid angle of view available to the camera. Due to spatial limitations this will probably also be about $2 \mathrm{~cm}$. Finally the desired resolution in radiative intensity is estimated to be $0.1 \mathrm{~W} / \mathrm{cm}^{3}$ through the line of sight. This example would produce a heat flux of $0.5 \mathrm{~mW} / \mathrm{cm}^{2}$ on the detector. This is very much in the range of current detectors which can measure fluxes as low as $1.0 \mu \mathrm{W} / \mathrm{cm}^{2}$.

The greatest concern for noise on the bolometer detectors comes from neutrons produced by the main plasma and the associated $\gamma$-rays. Estimates, made during the EDA phase of ITER, of neutron flux to bolometer detectors indicated neutron and $\gamma$-ray noise could reach the $0.5 \mathrm{~mW} / \mathrm{cm}^{2}$ 


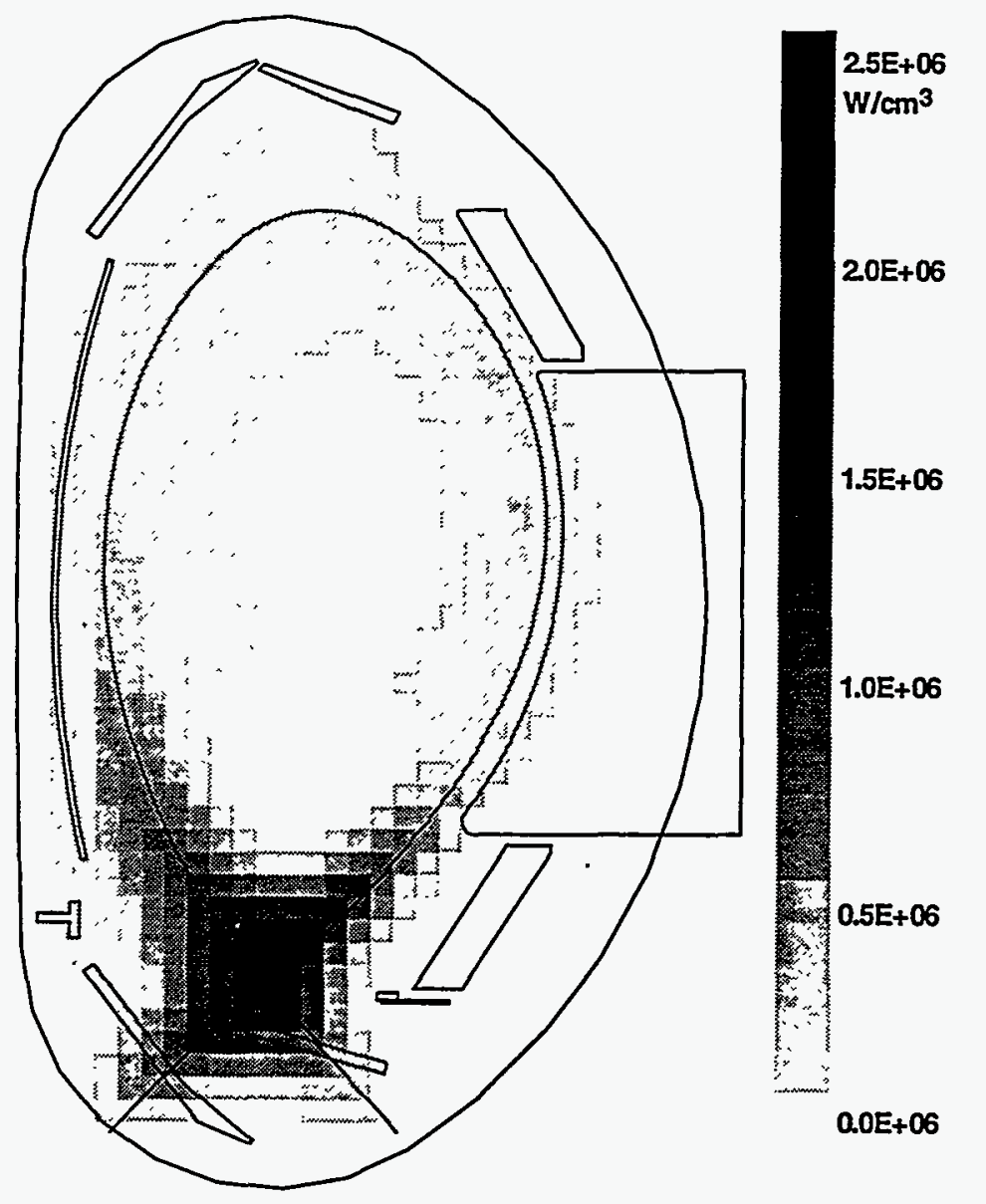

Fig. 7. An inversion of ASDEX-U bolometry data during neon injection experiments.

level. With the placement of detectors close to the divertor plasma and the additional shielding of the divertor from the main plasma neutrons this problem should be less in the current design. However, this issue does deserve further study.

Another concern is the neutral particle environment of the divertor. Neutral pressures of 5 mtorr to 100 mtorr can be expected in the divertor region ${ }^{1}$ where the bolometer cameras will be mounted. Neutral pressures this high affect the thermal characteristics of the bolometer sensors. If the gas is at wall temperature it will cool the active sensor imitating a lower incident radiation. For neutral gas at a higher temperature the opposite effect will take place. Since the sensors are enclosed in a camera structure with only a small viewing aperture for gas inlet, the gas should be at the temperature of the surrounding walls. This pressure effect might be taken into account, if it is known. It may be necessary to have a neutral pressure measurement in each of the cameras in order to keep an accurate calibration.

Higher energy neutrals, produced by charge-exchange or other ion-neutral collisions, may also be of some concern. A high energy neutral will deposit energy on the sensor just as well as a photon. However the neutrals represent a non-isotropic source, the hot neutrals directed toward the plasma are reionized where their energy remains in the plasma. This non-isotropic radiation still carries energy out of the plasma, but the inversion algorithms described above are no longer valid. If sufficient neutral gas is present between the source and the detector these particles may become thermalized before they strike the bolometer sensor. These processes will have to be modeled with an accurate divertor plasma before the magnitude of the problem can be identified.

\section{CONCLUSION}

A conceptual design for a divertor bolometer system for ITER has been sketched out. This design places the bolometer cameras close to the divertor plasma in the divertor structure. The proposed twelve cameras, six for each divertor leg, provide good coverage of the divertor and $\mathrm{X}$-point region. The specified spatial resolution of $5 \mathrm{~cm}$ is obtained by placing 20 channels in each camera. The signal levels for this geometry are adequate for current detector technology. However, the neutron and associated $\gamma$-ray noise will have to be studied to determine its magnitude. 


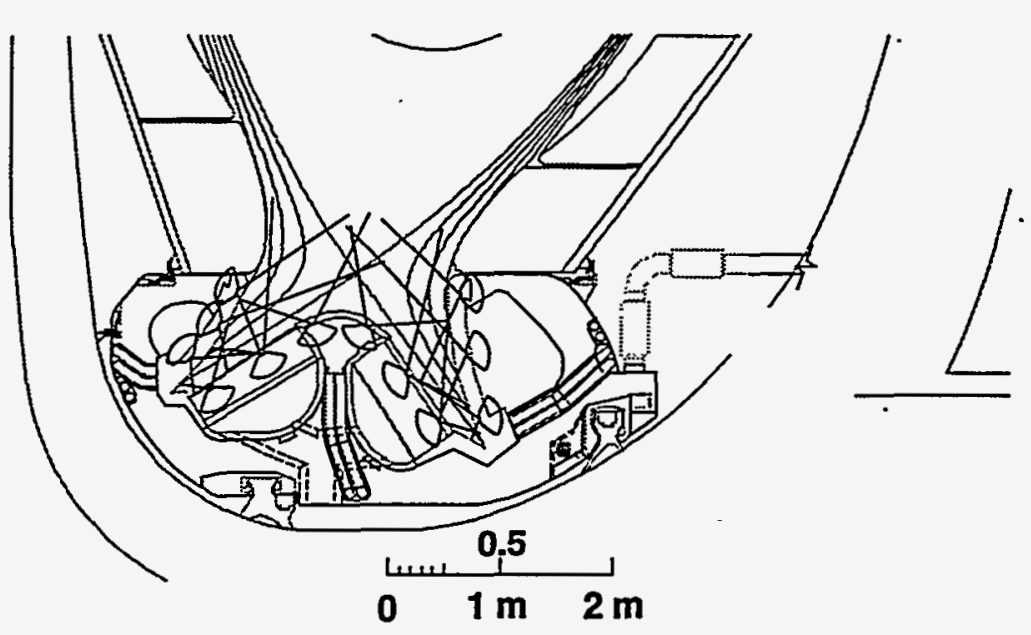

Fig. 8. A conceptual sketch of an ITER bolometer system. A total of six cameras are installed in each divertor leg with twenty detector channels in each camera.

If the bolometer cameras are moved back from the plasma and out of the divertor structure then the design will become much more difficult. From outside the divertor structure the signal levels will be much smaller if $5 \mathrm{~cm}$ spatial resolution is maintained. Also complete coverage of the divertor and $\mathrm{X}$-point plasma would require even greater apertures in the divertor structure. Finally, a bolometer system moved out of the divertor structure would not likely provide the number of views necessary for tomographic analysis of the spatial distribution of the radiation.

A successful bolometer system will be necessary not only for understanding the divertor, but for plasma control as well. For plasma control, eal-time processing of the bolometer sensor data will be needed. A linear combination of channels could produce any number of the divertor radiation characteristics. One combined signal could represent $\mathrm{X}$-point radiation, the distance from the divertor target of peak radiation, or signal that some surface is receiving too much radiative heating. These signals could then be used to control gas puffing from different regions, or change the pumping speed, as an example. The combination of signals could be produced by the statistical methods used on ASDEX-U, or possibly by neural network methods. However, a better understanding of divertor operation in general, and the ITER divertor in particular, is necessary before such control schemes can be envisioned.

\section{ACKNOWLEDGMENT}

This is a report of work supported by the U.S. Department of Energy under Contract No. DEAC03-89ER51114.

\section{REFERENCES}

${ }^{1}$ K.J. Dietz, et. al., Proc. 15th Intl. Conf on Plasma Phys. and Contr. Nucl. Fusion Research, 1994, Seville Spain IAEA-CN-60/E-1-I-4.

${ }^{2}$ A. Costley ITER Diagnostic Expert Group.

${ }^{3}$ K. F. Mast, et. al, Rev. Sci. Instrum. 63: 744 (1991).

4J. Goetz, et al., J. Nucl. Mater 220-222: 971-975 (1995).

${ }^{5}$ W.H. Press, B.P. Flannery, S.A. Teukolsky, W.T. Vetterling, "Numerical Recipes, the Art of Scientific Computing," Cambridge University Press 1986.

${ }^{6}$ A. W. Leonard, et. al., Rev. Sci.. Instrum. 66:1201 (1995).

7J.C. Fuchs, et. al., Proc of 21st EPS Conf. on Contr. Fusion and Plasma Physics, Montpellier (France).

${ }^{8} \mathrm{M}$. Marashek, to be submitted for publication. 